\title{
Stronger-correlated superconductivity in magic-angle twisted trilayer graphene
}

\section{Magic Angle Hierarchy in Twisted Graphene Multilayers}

Authors: E. Khalaf, A. J. Kruchkov, G. Tarnopolsky, and A. Vishwanath

arXiv:1901.10485, Aug 2019

2. Tunable Phase Boundaries and Ultra-Strong Coupling Superconductivity in Mirror Symmetric Magic-Angle Trilayer Graphene

Authors: JM Park, Y. Cao, K. Watanabe, T. Taniguchi, and P. Jarillo-Herrero

arXiv:2012.01434, Dec 2020

3. Electric field tunable unconventional superconductivity in alternating twist magic-angle trilayer graphene

Authors: Z Hao, A. M. Zimmerman, P. Ledwith, E. Khalaf, D.H. Najafabadi, K.

Watanabe, T. Taniguchi, A. Vishwanath and P. Kim

arXiv:2012.02773, Dec 2020

Recommended with a Commentary by Michael Zaletel, University
of California, Berkeley

Since the discovery of superconductivity in magic-angle twisted bilayer graphene (MATBG) featured in JCCM April 2018 (and revisited by the JCCM hopefully not one-toomany times since), strongly interacting phenomena have been discovered in a growing array of other "moire materials," from twisted bilayers of bilayers to ABC-stacked graphene on hBN. But despite this proliferation, only MA-TBG has displayed unambiguous and widely reproducible evidence for superconductivity. The featured experimental results on magicangle twisted trilayer graphene (MA-TTG) now add a new superconducting entry to the list. With the mechanism for superconductivity in MA-TBG still unresolved, comparison of these two materials offers some intriguing new constraints on which aspects of the physics we should be focusing on.

In the featured theory paper, Khalaf et al. first considered a graphene trilayer with a relative twist of $\theta$ between layers 1-2 and twist $-\theta$ between layers $2-3$, so that the whole structure has a mirror symmetry $M_{z}$ exchanging the two outer layers. The $M_{z}$ symmetry causes the spectrum to decouple into two sectors; the $M_{z}$-odd component has a Dirac spectrum with the velocity of graphene, while the two $M_{z}$-even states hybridize into a pair of flat-bands in exact analogy with MA-TBG. The rough picture is thus "TTG = MLG + TBG," and due to the high velocity and low DOS of the MLG-like Dirac spectrum, its effect on the interaction physics of the TBG-like flat-bands is expected to be rather small. 
The featured experiments of Park et. al. and Hao et. al. find that near the magic angle TTG indeed has a phase diagram which is quite similar to MATBG, with clear evidence for superconductivity when the system is doped away from electron filling $\nu=$ \pm 2 per moire cell. But MA-TTG has two interesting advantages over MA-TBG which give new evidence for strongly-coupled superconductivity (SC).

First, there is an effective $\sqrt{2}$-increase in the interlayer moire tunneling for the $M_{z}$-even sector, which correspondingly gives a $\sqrt{2}$-increase to the magic angle $\left(\theta_{T T G} \approx 1.56^{\circ}\right)$ and Coulomb scale $e^{2} / \epsilon L_{M}$. Due to these or other quantitative differences the experiments find a superconducting coherence length $\xi_{\mathrm{GL}}$ which is significantly smaller than in MA-TBG, approaching the inter-particle distance, evidence for superconductivity near the BEC-limit of the BEC-BCS

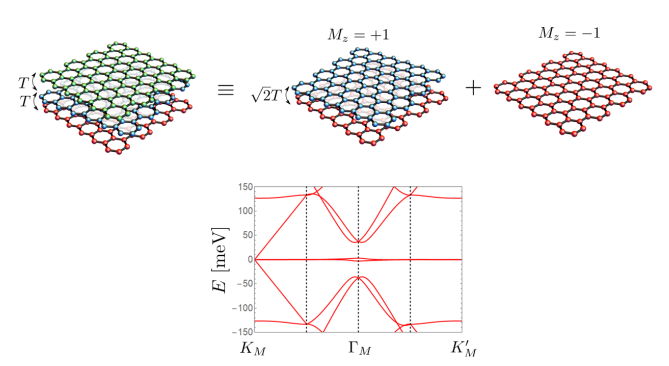

Figure 1: Twisted trilayer graphene, with a mirror symmetry $M_{z}$ relating the two out layers. $M_{z}$ odd and even sectors decouple, giving rise to a band structure formally equivalent to one copy of twisted bilayer graphene and a graphene monolayer. Fig. courtesy of E. Khalaf. crossover. Second, unlike MA-TBG, the band structure of MA-TTG is sensitive to the perpendicular displacement field $D$, which allows for in-situ tuning of the bandwidth at fixed density. The experiments make use of this tunability to map out the inter-relation between SC, symmetry-breaking, and van Hove singularities, and find that SC is not associated to regions of high-DOS - an apparent strike against a weak-coupling picture.

Using the temperature dependence of the critical field $B_{c}$ to extract the coherence length, both experiments find $\xi_{\mathrm{GL}} \approx 10-30 \mathrm{~nm}$ at optimal doping, depending on the precise criteria used to determine the critical temperature. Regardless of this factor of $3, \xi_{\mathrm{GL}}$ is small; the lattice scale is $L_{M} \approx 9 \mathrm{~nm}$, and the inter-particle distance between doped electrons is $d \approx 13 \mathrm{~nm}$. For comparison, in optimally doped MA-TBG $\xi_{\mathrm{GL}} \approx 50 \mathrm{~nm}$ (vs. MA-TTG's $30 \mathrm{~nm}$ ) at $L_{M}=12 \mathrm{~nm}$. As further evidence for strong-coupling, Park et al. define a Fermi temperature $T_{\mathrm{F}}$ by measuring the effective mass $m^{*}$ from quantum oscillations and assuming parabolic dispersion for the doped fraction of the electrons. With $T_{\mathrm{BKT}}$ the temperature of the $\mathrm{SC}$ transition, $T_{\mathrm{BKT}} / T_{\mathrm{F}}$ is then a proxy for the fraction of the electrons involved in superconductivity. At optimal doping Park et al. find MA-TTG obtains a value of $T_{\mathrm{BKT}} / T_{\mathrm{F}} \sim 0.1-0.125$, even larger than their finding of 0.05 (by the same measures) in the original MA-TBG report. For a single parabolic band, $T_{\mathrm{BKT}} / T_{\mathrm{F}}$ is upper-bounded by 0.125 at the BEC-BCS crossover, suggesting MA-TTG is approaching this limit. However, it is not clear we should ascribe any particularly special meaning to this numerical value since the flat bands are more complex than simple parabolas; for example several theoretical works have pointed out that due to band topology, even perfectly flat bands $\left(T_{F}=0\right)$ can support superconductivity with finite $T_{\mathrm{BKT}}$, in infinite violation of this bound. A violation of this form would be very interesting in its own right.

A second highlight of MA-TTG is the ability to distort the band structure using $D$ in order to discern what role the structure of the Fermi surface plays in superconductivity: is SC associated to regions of large DOS, as might be anticipated in weak-coupling approaches, or to some other feature? Using the low-field Hall density $n_{H}=-\left(e \frac{d R_{x y}}{d B}\right)^{-1}$ to track the Fermi 
volume, $n_{H}(n, D)$ is surveyed for three general features: low-DOS points (e.g. gaps or Dirac points); van-Hove singularities; and "resets" where (presumably) spontaneous symmetrybreaking causes discontinuous jumps in the Fermi volume. Across resets the near-integer difference $n_{H}-n$ can be used to infer the number of components frozen out by the symmetry breaking. Similar to the discovery of a "cascade" of transitions in MA-TBG, both experiments observe reset features in the vicinity of $\nu \sim-3$ and $\nu \sim-2$ across a range of $D$ (focusing on $\nu<0$ for simplicity). In the intervening region $-3 \lesssim \nu \lesssim-2$, the mismatch $\left|n_{H}-n\right| \approx 2$ indicates that only two flavor directions are active due to symmetry breaking. Across a wide range of $D$ the $\mathrm{SC}$ exists only in this presumptively symmetry-broken region. At high- $D$, Park et al. find that the SC-bounding resets intriguingly split into low-DOS and vHs-like features. Rather than SC centering on the high-DOS vHs, as might be expected in a weak-coupling picture, the strong $\mathrm{SC}$ is bounded by it, which is interpreted as further evidence for a strong-coupling limit.

It will be useful to understand the $D$-evolution more completely. A symmetry-breaking reset can either change or preserve the sign of $n_{H}$ depending on where the critical $n$ lies relative to the parent insulator. In the case where the reset would change sign, there may be some subtlety in distinguishing symmetry-breaking from a vHs. So perhaps the real message to take away here is "SC is bounded by symmetry-breaking," with the nature of the $\left|n_{H}-n\right| \approx 2$-component symmetry-broken state crucial to superconductivity.

It is tempting to infer some general trends across the growing array of graphene-based moire systems. At present, it appears that all the other graphene-based moire materials (twisted double-layer graphene, MATBG aligned on hBN, mono-bi, and ABC/hBN) show rich strongly-interacting behavior including spontaneous orbital ferromagnetism and zerofield quantized Hall effects, but they do not feature reproducible superconductivity. What distinguishes MATBG and MATTG from the rest? On the one hand, since their Hamiltonians are rather similar we might consider them co-dependent data points. But when $D$ breaks the $M_{z}$ symmetry (or when accounting for effects like relaxation), the quantitative details of the band-structure are quite different, while the SC persists.

There is however a universal feature which is uniquely common to them: only MATBG and MATTG feature 8 connected flat bands, while the others feature 4 . This 2-fold difference arises because the non-SC family does not have $C_{2 z} \mathcal{T}$ symmetry, which allows for a conventional band gap at neutrality, and hence the flat bands come in a spin-valley quartet which fill from $0<\nu<4$. In $\operatorname{MAT}(\mathrm{B} / \mathrm{T}) \mathrm{G}$ in contrast, $C_{2} \mathcal{T}$ symmetry is known to protect a band crossing at neutrality, and so there are eight flat bands which fill from $-4<\nu<4$. Thus, unique to these systems, we expect inter-band fluctuations in addition to iso-spin fluctuations, which may play an important role in mediating strongly-coupled SC.

A possible exception to this narrative is twisted bilayer graphene on a transition metal dichalcogenide (TMD) substrate, which "proximitizes" spin-orbit coupling into the TBG. Experiments at Caltech [1] find superconductivity down to angles $\theta \sim 0.9^{\circ}$, quite a bit below the magic angle and seen only when on the TMD substrate. It is not clear whether the effect of the TMD on TBG is dominated by Rashba SOC, which preserves $C_{2 z} \mathcal{T}$, or Ising SOC, which breaks it. A better microscopic understanding of this system, perhaps from ab-initio modeling, could provide an important data point on whether the $C_{2 z} \mathcal{T}$-protected crossing is a key ingredient for $\mathrm{SC}$, or temporary coincidence of a small $(N=7$ ?) sample size. 


\section{References}

[1] Superconductivity in metallic twisted bilayer graphene stabilized by WSe2, Arora, et al., Nature Vol 583, pg 379-384 (2020) 